\title{
The Existence and Uniqueness of Solutions for Mean-Reverting $\boldsymbol{\gamma}$-Process
}

\author{
Jiyuan Liao \\ School of Economic, Jinan University, Guangzhou, China \\ Email: liaojiyuan0805@163.com
}

How to cite this paper: Liao, J.Y. (2018) The Existence and Uniqueness of Solutions for Mean-Reverting $\gamma$-Process. Open Journal of Statistics, 8, 327-333.

https://doi.org/10.4236/ojs.2018.82021

Received: March 24, 2018

Accepted: April 21, 2018

Published: April 24, 2018

Copyright $\odot 2018$ by author and Scientific Research Publishing Inc. This work is licensed under the Creative Commons Attribution International License (CC BY 4.0).

http://creativecommons.org/licenses/by/4.0/ (c) (†) Open Access

\begin{abstract}
Empirical studies show that more and more short-term rate models in capturing the dynamics cannot be described by those classic ones. So the mean-reverting $\gamma$-process was correspondingly proposed. In most cases, its coefficients do not satisfy the linear growth condition; even they satisfy the local Lipschitz condition. So we still cannot examine its existence of solutions by traditional techniques. This paper overcomes these difficulties. Firstly, through using the function Lyapunov, it has proven the existence and uniqueness of solutions for mean-reverting $\gamma$-process when the parameter $\gamma \geq 1$. Secondly, when $\gamma \in\left[\frac{1}{2}, 1\right]$, it proves the solution is non-negative. Finally, it proves that there is a weak solution to the mean-reverting $\gamma$-process and the solution satisfies the track uniqueness by defining a function $\rho$. Therefore, the mean-reverting $\gamma$-process has the unique solution.
\end{abstract}

\section{Keywords}

Mean-Reverting $\gamma$-Process, Existence and Uniqueness, Non-Negative

\section{Introduction}

The short-term riskless interest rates started to develop from the late 1970s and gradually became one of the most fundamental and important quantities in financial markets. As the economy has evolved, many short-term interest rate models have also been proposed. Letting $S(t)$ represent the short-term interest rates, we have the following seven well-known models:

1) Merton [1]

$$
\mathrm{d} S(t)=\mu \mathrm{d} t+\sigma \mathrm{d} B(t)
$$

2) Vasicek [2] 


$$
\mathrm{d} S(t)=(\mu+\lambda S(t)) \mathrm{d} t+\sigma \mathrm{d} B(t)
$$

3) Geometric Brownian motion

$$
\mathrm{d} S(t)=\lambda S(t) \mathrm{d} t+\sigma S(t) \mathrm{d} B(t)
$$

4) Cox, Ingersoll and Ross [3]

$$
\mathrm{d} S(t)=(\mu+\lambda S(t)) \mathrm{d} t+\sigma \sqrt{S(t)} \mathrm{d} B(t) ;
$$

5) Brennan and Schwartz [4]

$$
\mathrm{d} S(t)=(\mu+\lambda S(t)) \mathrm{d} t+\sigma S(t) \mathrm{d} B(t) ;
$$

6) Cox, Ingersoll and Ross [5]

$$
\mathrm{d} S(t)=\sigma S^{\frac{3}{2}}(t) \mathrm{d} B(t) ;
$$

7) Constant Elasticity of Variance [6]

$$
\mathrm{d} S(t)=\lambda S(t) \mathrm{d} t+\sigma S^{\gamma}(t) \mathrm{d} B(t),
$$

where $\lambda, \mu$ and $\sigma$ are constants.

The above seven models can be combined with the following stochastic differential equation:

$$
\mathrm{d} S(t)=\lambda(\mu-S(t)) \mathrm{d} t+\sigma S^{\gamma}(t) \mathrm{d} B(t)
$$

by changing the parameters $\lambda, \mu$ and $\gamma$.

Where $\gamma=0$, we all know that the Equation (1.1) is the well-known Vasicek model [2]. If its coefficient $\lambda>0$, there is a mean-reverting property. In other words, the short-term interest rates $S(t)$ will approach the mean $\frac{\mu}{\lambda}$ in the long term. When $\gamma=1 / 2$, the Equation (1.1) is the well-known mean-reverting square root process [3]. Like the Vasicek model, the mean-reverting square root process also has a mean-reverting property. More importantly, it is a non-negative random process. So it is widely used to model volatility, interest rates and other financial quantities. When $\gamma \in[1 / 2,1]$, Mao et al. [7] discuss its analytical properties and strong convergence of numerical solutions. When $\gamma \geq 1$, it is a highly sensitive mean-reverting process, $\mathrm{Wu}$ et al. [8] show the way to prove that the solution of Equation (1.1) is existence and uniqueness.

In the next section, we first prove the existence and uniqueness of the solution of Equation (1.1) when $\gamma \geq 1$. In section three, we consider the existence and non-negative of the solution of Equation (1.1) when $\gamma \in[1 / 2,1]$. Finally, we will give the conclusion.

\section{The Unique Global Positive Solutions when $\gamma \geq 1$}

Throughout this paper, let $(\Omega, \mathcal{F}, \mathbb{P})$ be a complete probability space with a filtration $\left\{\mathcal{F}_{t}\right\}_{t \geq 0}$ satisfying the usual conditions. Let $B(t)$ be a scalar Brownian motion defined on the probability space. We consider the mean-reverting $\gamma$-process: 


$$
\mathrm{d} S(t)=\lambda(\mu-S(t)) \mathrm{d} t+\sigma S^{\gamma}(t) \mathrm{d} B(t)
$$

with the initial value $S(0)>0$, where $\lambda, \mu$ and $\sigma$ are positive and $\gamma \geq 1$.

Theorem 2.1. For any given initial value $S(0)>0, \lambda, \mu$ and $\sigma>0$, there exists a unique positive global solution $S(t)$ to Equation (2.1) on $t \geq 0$.

Proof: Clearly, the coefficients of (2.1) satisfy the local Lipchitz condition. Therefore, for any given initial value $S(0)>0$, there must exist a unique local solution $S(t) \in\left[0, \tau_{e}\right)$, where $\tau_{e}$ is the explosion time. To show the solution is global, we next show that $\tau_{e}=\infty$ a.s.

Firstly, we choose a sufficient large integer $k_{0}>0$, namely $S(0) \in\left(1 / k_{0}, k_{0}\right)$, and then we define the following stopping time,

$$
\tau_{k}=\inf \left\{t \in\left[0, \tau_{e}\right]: S(t) \notin\left[\frac{1}{k}, k\right]\right\}, k \geq k_{0} .
$$

Let $\inf \varnothing=\infty$, it is clear that $\tau_{k}$ is increasing as $k \rightarrow \infty$. Set $\tau_{\infty}=\lim _{k \rightarrow \infty} \tau_{k}$, we get $\tau_{\infty} \leq \tau_{e}$ a.s. To complete the proof what we need to show is that $\tau_{\infty}=\infty$ a.s. To prove this result, for any constant $T$, if there is $P\left(\tau_{k} \leq T\right) \rightarrow 0, k \rightarrow \infty$, namely $\lim _{k \rightarrow \infty} E\left[I_{\left(\tau_{k} \leq T\right)}\right]=0$, by the control of convergence theorem, then we can get $E\left[I_{\left(\tau_{\infty} \leq T\right)}\right]=0$, so there is $P\left(\tau_{\infty}=\infty\right)=1$, which is the result we need.

For $m \in(0,1)$, we define a $C^{2}$-function $V:(0, \infty) \rightarrow(0, \infty)$ by

$$
V(S)=S^{m}-1-m \ln S \text {. }
$$

It is easy to show that $V(S) \geq 0$ and $V(S) \rightarrow \infty$ as $S \rightarrow 0$ or $S \rightarrow \infty$. Apply the Itô formula yields

$$
\begin{aligned}
\mathrm{d} V(S)= & \frac{\partial V(S)}{\partial t} \mathrm{~d} t+\frac{\partial V(S)}{\partial S} \mathrm{~d} S+\frac{1}{2} \frac{\partial^{2} V(S)}{\partial S^{2}}(\mathrm{~d} S)^{2} \\
= & \left(m S^{m-1}-\frac{m}{S}\right) \mathrm{d} S+\frac{1}{2}\left[m(m-1) S^{m-2}+\frac{m}{S^{2}}\right](\mathrm{d} S)^{2} \\
= & \left\{\lambda m\left(\mu S^{m-1}-S^{m}-\mu S^{-1}+1\right)+\frac{m \sigma^{2}}{2}\left[(m-1) S^{m+2(\theta-1)}+S^{2(\theta-1)}\right]\right\} \mathrm{d} t \\
& +\sigma m\left(S^{m+\theta-1}-S^{\theta-1}\right) \mathrm{d} B(t)
\end{aligned}
$$

For $m \in(0,1)$, by the boundedness of polynomial, it is easy to show that there is a constant $K_{1}$ such that

$$
\lambda m\left(\mu S^{m-1}-S^{m}-\mu S^{-1}+1\right)+\frac{m \sigma^{2}}{2}\left[(m-1) S^{m+2(\theta-1)}+S^{2(\theta-1)}\right] \leq K_{1} .
$$

Therefore, for $\forall t \in[0, T]$,

$$
\mathrm{d} V(S) \leq K_{1} \mathrm{~d} t+\sigma m\left(S^{m+\theta-1}-S^{\theta-1}\right) \mathrm{d} B(t), \quad S \in(0, \infty)
$$

On the both sides of the formula, we take integrate from 0 to $\tau_{k} \wedge T$. And then taking expectations, we have

$$
E V\left[S\left(\tau_{k} \wedge T\right)\right] \leq V[S(0)]+K_{1} E\left(\tau_{k} \wedge T\right) \leq V[S(0)]+K_{1} T .
$$

In the following, we use the proof by contradiction to show. If the previous 
conclusion is wrong, then there exists $T>0$ and $\varepsilon \in(0,1)$, such that

$$
P\left(\tau_{\infty} \leq T\right)>\varepsilon .
$$

So there is an integer $K_{1} \geq K_{0}$,

$$
P\left(\tau_{K} \leq T\right) \geq \varepsilon, \quad \forall K \geq K_{1} .
$$

Due to $\tau_{\infty}=\lim _{k \rightarrow 0} \tau_{k}$, then there exists an integer $K_{1} \geq K_{0}$, we get $\left|\tau_{k}-\tau_{\infty}\right|<\varepsilon_{1}$ as $K \geq K_{1}$. In addition, since $\left\{\tau_{k}\right\}$ is monotonically increasing, we can obtain $\tau_{k}<\tau_{\infty}-\varepsilon_{1}$. Set $\Omega_{k}=\left\{\tau_{K} \leq T\right\}$, from the above formula, we get $P\left(\Omega_{K}\right) \geq \varepsilon$ as $K \geq K_{1}$. For every $\omega \in \Omega_{K}$, there is $S\left(\tau_{k}, \omega\right) \geq k$ or $S\left(\tau_{k}, \omega\right) \leq \frac{1}{k}$. It is easy to show that the function $V(S)$ monotonically decreases in the interval $(0,1)$, monotonically increases in the interval $(1,+\infty)$. So

$$
V\left[S\left(\tau_{K}, \omega\right)\right] \geq V(k) \wedge V\left(\frac{1}{k}\right) .
$$

We have

$$
V[S(0)]+K_{1} T \geq E\left[I_{\Omega_{K}(\omega)} \cdot V\left(S\left(\tau_{k}, \omega\right)\right)\right]=P\left(\Omega_{K}\right) \cdot\left[V(K) \wedge V\left(\frac{1}{K}\right)\right] .
$$

Due to $V(K) \wedge V\left(\frac{1}{K}\right) \rightarrow \infty$, we have $P\left(\Omega_{K}\right) \rightarrow 0$ as $K \rightarrow \infty$. This implies $P\left(\tau_{\infty}=\infty\right)=1$. We finish the proof.

\section{The Unique Positive Solutions when $\gamma \in[1 / 2,1]$}

When $\gamma \in[1 / 2,1]$, in order to prove the conclusion, we complete in two steps. The first is to prove the non-negative solution if the solution exists. The second is to prove the existence and uniqueness of solutions.

\subsection{Non-Negative Solution}

As mentioned earlier, like the well-known mean-reverting square root process [5], it is widely used to model volatility, interest rates and other financial quantities. But there is a premise that is non-negative. So we will prove it is a non-negative solution.

We consider the mean-reverting $\gamma$-process:

$$
\mathrm{d} S(t)=\lambda(\mu-S(t)) \mathrm{d} t+\sigma S^{\gamma}(t) \mathrm{d} B(t)
$$

with the initial value $S(0)>0$, where $\lambda, \mu$ and $\sigma$ are positive and $\gamma \in[1 / 2,1]$.

Lemma 3.1. For given any initial data $S(0)=S_{0}>0$, the solution $S(t)$ of Equation (3.1) will never become negative with probability 1 .

Proof: We consider the following equation

$$
\mathrm{d} S(t)=\lambda(\mu-S(t)) \mathrm{d} t+\sigma|S(t)|^{\gamma} \mathrm{d} B(t), \quad t \geq 0 .
$$

It is clear that the solution of Equation (3.2) is equivalent to the statement of 
the lemma 3.1.

In the following, we will show that the solution of Equation (3.2) will never become negative with probability 1 for any initial data $S(0)=S_{0}>0$.

Let $a_{0}=1$, and for each integer $k=1,2, \cdots$,

$$
a_{k}= \begin{cases}\mathrm{e}^{-k(k+1)} & \text { if } \theta=\frac{1}{2}, \\ {\left[\frac{(2 \theta-1) k(k+1)}{2}\right]^{1 /(1-2 \theta)}} & \text { if } \frac{1}{2}<\theta \leq 1,\end{cases}
$$

So it is easy to get the following result:

$$
\int_{a_{k}}^{a_{k-1}} \frac{1}{u^{2 \theta}} \mathrm{d} u=k .
$$

For every $k=1,2, \cdots$, there clearly exists a continuous function $\psi_{k}(u)$ with support in $\left(a_{k}, a_{k-1}\right)$ such that

$$
0 \leq \psi_{k}(u) \leq \frac{2}{k u^{2 \theta}} \quad \text { for } a_{k}<u<a_{k-1}
$$

and $\int_{a_{k}}^{a_{k-1}} \psi_{k}(u) \mathrm{d} u=1$.

Define

$$
\varphi_{k}= \begin{cases}0 & \text { for } x \geq 0, \\ \int_{0}^{-x} \mathrm{~d} y \int_{0}^{y} \psi_{k}(u) \mathrm{d} u & \text { for } x<0 .\end{cases}
$$

Then $\varphi_{k} \in C^{2}(\mathbb{R}, \mathbb{R})$ and has the following properties:

1) $-1 \leq \varphi_{k}^{\prime}(x) \leq 0$ for $a_{k}<x<a_{k-1}$, or otherwise $\varphi_{k}^{\prime}(x)=0$;

2) $\left|\varphi_{k}^{\prime \prime}(x)\right| \leq \frac{2}{k|x|^{2 \theta}}$ for $a_{k}<x<a_{k-1}$, or otherwise $\varphi_{k}^{\prime \prime}(x)=0$;

3) $|x|-a_{k-1} \leq \varphi_{k}(x) \leq|x|$ for $\forall x \in \mathbb{R}$.

It is easy to show the properties (1), (2) and (3).

Now for $\forall t \geq 0$, apply the Itô formula yields

$$
\begin{aligned}
\varphi_{k}(S(t))= & \varphi_{k}(S(0))+\int_{0}^{t}\left[\lambda(\mu-S(u)) \varphi_{k}^{\prime}(S(u))+\frac{\sigma^{2}}{2}|S(u)|^{2 \theta} \varphi_{k}^{\prime \prime}(S(u))\right] \mathrm{d} u \\
& +\sigma \int_{0}^{t} \varphi_{k}(S(u))|S(u)|^{\theta} \mathrm{d} B(u)
\end{aligned}
$$

Then taking expectations in both sides, we have

$$
\begin{aligned}
\mathrm{E} \varphi_{k}(S(t)) & =\varphi_{k}(S(0))+\mathrm{E} \int_{0}^{t}\left[\lambda(\mu-S(u)) \varphi_{k}^{\prime}(S(u))+\frac{\sigma^{2}}{2}|S(u)|^{2 \theta} \varphi_{k}^{\prime \prime}(S(u))\right] \mathrm{d} u \\
& \leq \mathrm{E} \int_{0}^{t} \frac{\sigma^{2}}{2}|S(u)|^{2 \theta} \frac{2}{k|S(u)|^{2 \theta}} \mathrm{d} u=\frac{\sigma^{2} t}{k}
\end{aligned}
$$

Therefore, apply the properties (3), we have

$$
-a_{k-1} \leq \mathrm{ES}^{-}(t)-a_{k-1} \leq \frac{\sigma^{2} t}{k},
$$


where

$$
S^{-}(t)= \begin{cases}-S(t) & \text { if } S(t)<0, \\ 0 & \text { if } S(t) \geq 0 .\end{cases}
$$

Letting $k \rightarrow \infty$, we get that $\mathrm{ES}^{-}(t) \leq a_{k-1}+\frac{\sigma^{2} t}{k} \rightarrow 0$. So we have $\mathrm{ES}^{-}(t)=0$ for $\forall t \geq 0$. This implies that the Lemma 3.1 is true.

\subsection{The Existence and Uniqueness of Solutions}

The following result shows that the solution $S(t)$ of Equation (3.1) is exist and unique.

Theorem 3.1. For any given initial value $S(0)>0, \lambda, \mu$ and $\sigma>0$, there exists a unique solution $S(t)$ to Equation (3.1) on $t \geq 0$.

Proof: Clearly, the coefficients of (3.1) satisfy the linear growth condition. From I. Karatzas and S.E. Shreve [9], we know that there is a weak solution for Equation (3.1). Next, we will show that the solution satisfies the track uniqueness.

It is easy to show that the following inequality is right:

$$
|x+y|^{\gamma} \leq x^{\gamma}+y^{\gamma}
$$

with the $\gamma \in[1 / 2,1]$.

Thus, we have

$$
|(x-y)+y|^{\gamma} \leq|x-y|^{\gamma}+y^{\gamma},
$$

which implies

$$
x^{\gamma}-y^{\gamma} \leq|x-y|^{\gamma} .
$$

Similarly, the following inequality can be proved

$$
y^{\gamma}-x^{\gamma} \leq|x-y|^{\gamma} .
$$

So we have

$$
\left|x^{\gamma}-y^{\gamma}\right| \leq|x-y|^{\gamma}
$$

Define

$$
\rho(u)=\sigma u^{\gamma}, \quad 1 / 2 \leq \gamma \leq 1 .
$$

It is easy to get the following result:

$$
\begin{gathered}
\rho(0)=0, \\
\int_{0+} \rho^{-2}(u) \mathrm{d} u=\int_{0+} \frac{1}{\sigma^{2} u^{2 \gamma}} \mathrm{d} u=\infty .
\end{gathered}
$$

By the diffusion coefficient of Equation (3.1), we get

$$
\left|\sigma x^{\gamma}-\sigma y^{\gamma}\right| \leq \sigma|x-y|^{\gamma}=\rho(|x-y|) .
$$

Referring to the theorem 19.5 of The Elements of Stochastic Analysis [10], the 
theorem 3.1 is true. Therefore, the solution $S(t)$ of Equation (3.1) is exist and unique. We finish the proof.

\section{Conclusion}

In this article, we proved the existence and uniqueness of the solution of Equation (1.1) when $\gamma \geq 1$. And we get the existence and non-negative of the solution of Equation (1.1) when $\gamma \in[1 / 2,1]$. Further, we can study the solution of the Equation (1.1) when $\gamma \in[0,1 / 2]$.

\section{References}

[1] Merton, R.C. (1973) Theory of Rational Option Pricing. The Bell Journal of Economics and Management Science, 4, 141-183.

[2] Vasicek, O. (1977) An Equilibrium Characterization of the Term Structure. Journal of Financial Economics, 5, 177-188.

[3] Cox, J.C., Ingersoll Jr., J.E. and Ross, S.A. (1985) A Theory of the Term Structure of Interest Rates. The Economist, 53, 385-407.

[4] Brennan, M.J. and Schwartz, E.S. (1980) Analyzing Convertible Bonds. Journal of Financial and Quantitative Analysis, 15, 907-929.

[5] Cox, J.C., Ingersoll Jr., J.E. and Ross, S.A. (1980) An Analysis of Variable Rate Loan Contracts. Journal of Finance, 35, 389-403.

https://doi.org/10.1111/j.1540-6261.1980.tb02169.x

[6] Cox, J.C. (1975) Notes on Option Pricing I: Constant Elasticity of Variance Diffusion. Working Paper, Stanford University.

[7] Mao, X., Truman, A. and Yuan, C. (2006) Euler-Maruyama Approximations in Mean-Reverting Stochastic Volatility Model under Regime-Switching. Journal of Applied Mathematics and Stochastic Analysis, 2, 1-20.

[8] Wu, F., Mao, X. and Chen, K. (2008) A Highly Sensitive Mean-Reverting Process in Finance and the Euler-Maruyama Approximations. Journal of Mathematical Analysis and Applications, 348, 540-554. https://doi.org/10.1016/j.jmaa.2008.07.069

[9] Karatzas, I. and Shreve, S.E. (1991) Brownian Motion and Stochastic Calculus. Springer.

[10] Huang, Z.Y. (2001) The Elements of Stochastic Analysis. Science. 